\title{
Asymptotic Bounds for the Time-Periodic Solutions to the Singularly Perturbed Ordinary Differential Equations
}

\author{
Gabil M. Amiraliyev and Aysenur Ucar \\ Department of Mathematics, Sinop University, 57000 Sinop, Turkey \\ Correspondence should be addressed to Aysenur Ucar; aysnrucar@gmail.com
}

Received 3 October 2013; Accepted 24 October 2013

Academic Editors: F. Mukhamedov, G. Tsiatas, and H. Yang

Copyright (c) 2013 G. M. Amiraliyev and A. Ucar. This is an open access article distributed under the Creative Commons Attribution License, which permits unrestricted use, distribution, and reproduction in any medium, provided the original work is properly cited.

\begin{abstract}
The periodical in time problem for singularly perturbed second order linear ordinary differential equation is considered. The boundary layer behavior of the solution and its first and second derivatives have been established. An example supporting the theoretical analysis is presented.
\end{abstract}

\section{Introduction and Preliminaries}

In this paper we investigate the equation

$$
L u \equiv \varepsilon u^{\prime \prime}+a(t) u^{\prime}+b(t) u=f(t), \quad 0<t<T,
$$

with the periodic conditions

$$
u(0)=u(T), \quad u^{\prime}(0)=u^{\prime}(T),
$$

where $\varepsilon \in(0,1]$ is the perturbation parameter, $0<\alpha \leq a(t) \leq$ $a^{*}, 0<\beta \leq b(t) \leq b^{*}$, and $f(t)$ are the $T$-periodic functions satisfying $a, b, f \in C^{1}[0, T]$.

Periodical in time problems arise in many areas of mathematical physics and fluid mechanics [1-3]. Various properties of periodical in time problems in the absence of boundary layers have been investigated earlier by many authors (see, e.g., $[4,5]$ and references therein).

The qualitative analysis of singular perturbation situations has always been far from trivial because of the boundary layer behavior of the solution. In singular perturbation cases, problems depend on a small parameter $\varepsilon$ in such a way that the solution exhibits a multiscale character; that is, there are thin transition layers where the solution varies rapidly while away from layers and it behaves regularly and varies slowly [6-8].

We note that periodical in space variable problems and also their approximate solutions were investigated by many authors (see, e.g., [9-13]).
In this note we establish the boundary layer behaviour for $u(t)$ of the solution of (1)-(2) and its first and second derivatives. The maximum principle, which is usually used for periodical boundary value problems, is not applicable here; because of this we use another approach which is convenient for this type of problems. The approach used here is similar to those in $[9,14,15]$.

Note 1. Throughout the paper $C$ denotes the generic positive constants independent of $\varepsilon$. Such a subscripted constant is also independent of $\varepsilon$, but its value is fixed.

Lemma 1. Let $\delta(t) \geq 0$ be the continuous function defined on $[0, T]$ and $c_{0}(t), \rho(t) \in C[0, T]$ and $\gamma, \mu$ are given constants. If

$$
\delta^{\prime}(t)+c_{0}(t) \delta(t) \leq \rho(t), \quad \delta(0) \leq \mu \delta(T)+\gamma,
$$

then

$$
\begin{aligned}
\delta(t) \leq & \left(1-\mu e^{-\int_{0}^{T} c_{0}(s) d s}\right)^{-1} \\
& \times\left(\gamma e^{-\int_{0}^{T} c_{0}(\eta) d \eta}+\int_{0}^{T} \rho(s) e^{-\int_{s}^{T} c_{0}(\eta) d \eta} d s\right) e^{-\int_{0}^{T} c_{0}(\eta) d \eta} \\
& +\int_{0}^{t} \rho(s) e^{-\int_{s}^{T} c_{0}(\eta) d \eta} d s
\end{aligned}
$$


provided that

$$
1-\mu e^{-\int_{0}^{T} c_{0}(s) d s}>0
$$

Proof. Inequality (4) can be easily obtained by using first order differential inequality containing initial condition.

\section{Asymptotic Estimate}

We now give a priori bounds on the solution and its derivatives for problem (1)-(2).

Theorem 2. The solution $u(t)$ of the problem (1)-(2) satisfies the bound

$$
\varepsilon\left|u^{\prime}\right|^{2}+|u|^{2} \leq C \int_{0}^{t}|f(s)|^{2} d s
$$

provided that

$$
\gamma=\lambda_{0} \min _{[0, T]}\left(2 b(t)-a^{\prime}(t)\right)-\overline{b^{*}}>0,
$$

where

$$
\overline{b^{*}}=\max _{[0, T]} b^{\prime}(t), \quad 0<\lambda_{0}<\frac{\left(\alpha+\sqrt{\alpha^{2}+8 \beta}\right)}{4} .
$$

Proof. Consider the identity

$$
L u\left(u^{\prime}+\lambda u\right)=\left(u^{\prime}+\lambda u\right) f(t)
$$

with parameter $\lambda>0$ which will be chosen later. By using the equalities

$$
\begin{gathered}
\varepsilon u^{\prime \prime} u^{\prime}=\frac{\varepsilon}{2}\left[\left(u^{\prime}\right)^{2}\right]^{\prime}, \\
\lambda u^{\prime \prime} u=\lambda\left(u^{\prime} u\right)^{\prime}-\lambda\left(u^{\prime}\right)^{2}, \\
\lambda a(t) u^{\prime} u=\frac{\lambda}{2} a(t)\left(u^{2}\right)^{\prime}=\frac{\lambda}{2}\left[a(t) u^{2}\right]^{\prime}-\frac{\lambda}{2} a^{\prime}(t) u^{2}, \\
b(t) u^{\prime} u=\frac{1}{2} b(t)\left(u^{2}\right)^{\prime}=\frac{1}{2}\left[b(t) u^{2}\right]^{\prime}-\frac{1}{2} b^{\prime}(t) u^{2},
\end{gathered}
$$

and the inequalities

$$
\begin{aligned}
& u^{\prime} f(t) \leq \mu_{1}\left(u^{\prime}\right)^{2}+\frac{1}{4 \mu_{1}} f^{2}(t), \quad \mu_{1}>0, \\
& \lambda u f(t) \leq \lambda \mu_{2} u^{2}+\frac{\lambda}{4 \mu_{2}} f^{2}(t), \quad \mu_{2}>0,
\end{aligned}
$$

in (9), we have

$$
\begin{aligned}
\left\{\varepsilon u^{\prime 2}+\right. & \left.2 \varepsilon \lambda u^{\prime} u+\lambda a(t) u^{2}+b(t) u^{2}\right\}^{\prime} \\
\leq & -2\left\{a(t)-\varepsilon \lambda-\mu_{1}\right\} u^{\prime 2} \\
& +\left\{b^{\prime}(t)+\lambda a^{\prime}(t)-2 \lambda b(t)+2 \lambda \mu_{2}\right\} u^{2} \\
& +\left\{\frac{1}{2 \mu_{1}}+\frac{\lambda}{2 \mu_{2}}\right\} f^{2}(t) .
\end{aligned}
$$

Denoting now $\delta(t)=\varepsilon u^{\prime 2}+2 \varepsilon \lambda u^{\prime} u+\lambda a(t) u^{2}+b(t) u^{2}$ and choosing $\mu=1 / 2$, we arrive at

$$
\delta(t) \geq \frac{\varepsilon}{2} u^{\prime 2}+\{\beta+\lambda(\alpha-2 \lambda \varepsilon)\} u^{2} .
$$

After taking $\lambda=\lambda_{0}<\left(\alpha+\sqrt{\alpha^{2}+8 \beta}\right) / 4$, the last inequality reduces to

$$
\delta(t) \geq C_{0}\left(\varepsilon u^{\prime 2}+u^{2}\right)
$$

where

$$
0<C_{0}=\min \left\{\frac{1}{2}, \beta+\lambda_{0}\left(\alpha-2 \lambda_{0} \varepsilon\right)\right\} .
$$

On the other hand for the function $\delta(t)$ holds the following inequality clearly:

$$
\begin{aligned}
\delta(t) & \leq \varepsilon(1+\lambda) u^{\prime 2}+\left(b^{*}+\varepsilon \lambda+\lambda a^{*}\right) u^{2} \\
& \leq \varepsilon\left(1+\lambda_{0}\right) u^{\prime 2}+\left(b^{*}+\lambda_{0}+\lambda_{0} a^{*}\right) u^{2} .
\end{aligned}
$$

For the right-hand side of inequality (12), we have

$$
\begin{aligned}
2\{a(t) & \left.-\varepsilon \lambda-\mu_{1}\right\} u^{\prime 2} \\
& +\left\{-b^{\prime}(t)-\lambda a^{\prime}(t)+2 \lambda b(t)-2 \lambda \mu_{2}\right\} u^{2} \\
\geq & 2 \varepsilon\left\{\alpha-\varepsilon \lambda_{0}-\mu_{1}\right\} u^{\prime 2} \\
& +\left\{-\overline{b^{*}}-\lambda_{0} a^{\prime}(t)+2 \lambda_{0} b(t)-2 \lambda_{0} \mu_{2}\right\} u^{2} \\
\geq & \alpha \varepsilon u^{\prime 2}+\frac{\gamma}{2} u^{2} .
\end{aligned}
$$

Taking into account $\varepsilon \leq 1$ and $\gamma>0$, after choosing $\mu_{1}=$ $\left(\alpha-2 \lambda_{0}\right) / 2$ and $\mu_{2}=\gamma / 4 \lambda_{0}$, we have

$$
\delta^{\prime}(t) \leq-C_{1} \delta(t)+\rho(t), \quad \delta(0)=\delta(T),
$$

where

$$
0<C_{1}=\min \left\{\frac{\alpha}{1+\lambda_{0}}, \frac{\gamma}{2\left(b^{*}+\lambda_{0}+\lambda_{0} a^{*}\right)}\right\},
$$

$$
\rho(t)=\left\{\frac{1}{2 \mu_{1}}+\frac{\lambda}{2 \mu_{2}}\right\} f^{2}(t)
$$

From (18) by using Lemma 1, we have

$$
\delta(t) \leq \frac{e^{-C_{1} t}}{1-e^{-C_{1} T}} \int_{0}^{T} \rho(s) e^{-C_{1}(t-s)} d s+\int_{0}^{t} \rho(s) e^{-C_{1}(t-s)} d s
$$

which proves Theorem 2.

Note 2. As it is seen from (6)

$$
|u(t)| \leq C\|f\|_{2},
$$

where

$$
\|f\|_{2}=\int_{0}^{T}\left|f^{2}(s)\right|^{1 / 2} d s .
$$


Theorem 3. Under the assumptions of Theorem 2, the following asymptotic estimates for the derivatives hold true:

$$
\left|u^{(k)}(t)\right| \leq C\left\{1+\varepsilon^{1-k} e^{-\alpha t / \varepsilon}\right\}, \quad 0 \leq t \leq T, k=0,1,2 .
$$

Proof. The case $k=0$ directly follows from the identity (4).

For $k=1$, the problem (1)-(2) can be rewritten as

$$
\begin{gathered}
\varepsilon u^{\prime \prime}+a(t) u^{\prime}=F(t), \quad 0<t<T, \\
|u(0)| \leq C, \\
u^{\prime}(0)=u^{\prime}(T),
\end{gathered}
$$

where

$$
F(t)=f(t)-b(t) u
$$

and by virtue of Theorem 2

$$
|F(t)| \leq C .
$$

The solution of (24)-(26) can be expressed as

$$
u^{\prime}(t)=u^{\prime}(0) e^{-(1 / \varepsilon) \int_{0}^{t} a(s) d s}+\frac{1}{\varepsilon} \int_{0}^{t} F(s) e^{-(1 / \varepsilon) \int_{s}^{t} a(\xi) d \xi} d s,
$$

and taking into account (26), we have

$$
u^{\prime}(0)=\left(1-e^{-(1 / \varepsilon) \int_{0}^{T} a(s) d s}\right)^{-1} \frac{1}{\varepsilon} \int_{0}^{T} F(s) e^{-(1 / \varepsilon) \int_{s}^{T} a(\xi) d \xi} d s .
$$

Thus we get

$$
\left|u^{\prime}(0)\right| \leq \frac{C \alpha^{-1}\left(1-e^{-\alpha T / \varepsilon}\right)}{1-e^{-a^{*} T / \varepsilon}} \leq C \alpha^{-1} .
$$

The relation (29) along with (31) leads to (23) for $k=1$ immediately.

Next for $k=2$, from (1) we have

$$
\left|u^{\prime \prime}(0)\right|=\frac{1}{\varepsilon}\left|f(0)-b(0) u(0)-a(0) u^{\prime}(0)\right| \leq \frac{C}{\varepsilon} .
$$

Differentiating now (1), we obtain

$$
\varepsilon u^{\prime \prime \prime}+a(t) u^{\prime \prime}=f^{\prime}(t)-b^{\prime}(t) u-b(t) u^{\prime}-a^{\prime}(t) u \equiv \varphi(t) .
$$

Under the smoothness conditions on data functions and boundness of $u(t)$ and $u^{\prime}(t)$, we deduce evidently

$$
|\varphi(t)| \leq C \text {. }
$$

The solution of (33) is

$$
u^{\prime \prime}(t)=u^{\prime \prime}(0) e^{-(1 / \varepsilon) \int_{0}^{t} a(s) d s}+\frac{1}{\varepsilon} \int_{0}^{t} \varphi(s) e^{-(1 / \varepsilon) \int_{s}^{t} a(\xi) d \xi} d s .
$$

The validity of (23) for $k=2$ now easily can be seen by using (32) $-(34)$ in (35).

\section{Example}

Consider the particular problem with

$$
a(t)=4, \quad b(t)=3, \quad f(t)=3 t, \quad T=1 .
$$

The solution of this problem is given by

$$
u(t)=A_{1} e^{-((2-\sqrt{4-3 \varepsilon}) / \varepsilon) t}+A_{2} e^{-((2+\sqrt{4-3 \varepsilon}) / \varepsilon) t}+t-\frac{4}{3},
$$

where

$$
\begin{aligned}
& A_{1}=\frac{2+\sqrt{4-3 \varepsilon}}{2 \sqrt{4-3 \varepsilon}\left(1-e^{-(2-\sqrt{4-3 \varepsilon}) / \varepsilon}\right)}, \\
& A_{2}=\frac{\sqrt{4-3 \varepsilon}-2}{2 \sqrt{4-3 \varepsilon}\left(1-e^{-(2+\sqrt{4-3 \varepsilon}) / \varepsilon}\right)} .
\end{aligned}
$$

For the first derivative we have

$$
\begin{aligned}
u^{\prime(t)}= & -\frac{3}{2 \sqrt{4-3 \varepsilon}\left(1-e^{-(2-\sqrt{4-3 \varepsilon}) / \varepsilon}\right)} e^{-((2-\sqrt{4-3 \varepsilon}) / \varepsilon) t} \\
& -\frac{3}{2 \sqrt{4-3 \varepsilon}\left(1-e^{-(2+\sqrt{4-3 \varepsilon}) / \varepsilon}\right)} e^{-((2+\sqrt{4-3 \varepsilon}) / \varepsilon) t}+1
\end{aligned}
$$

from which it is clear that the first derivative of $u(t)$ is uniformly bounded but has a boundary layer near $t=0$ of thickness $O(\varepsilon)$.

The second derivative

$u^{\prime \prime}(t)$

$$
\begin{aligned}
=-\frac{3}{\varepsilon}( & \frac{2-\sqrt{4-3 \varepsilon}}{2 \sqrt{4-3 \varepsilon}\left(1-e^{-(2-\sqrt{4-3 \varepsilon}) / \varepsilon}\right)} e^{-((2-\sqrt{4-3 \varepsilon}) / \varepsilon) t} \\
& \left.+\frac{2+\sqrt{4-3 \varepsilon}}{2 \sqrt{4-3 \varepsilon}\left(1-e^{-(2+\sqrt{4-3 \varepsilon}) / \varepsilon}\right)} e^{-((2+\sqrt{4-3 \varepsilon}) / \varepsilon) t}\right)
\end{aligned}
$$

is unbounded while $\varepsilon$ values are tending to zero.

Therefore we observe here the accordance in our theoretical results described above.

\section{References}

[1] E. Chadwick and R. El-Mazuzi, "A coupled far-field formulation for time-periodic numerical problems in fluid dynamics," Proceedings of the Indian Academy of Sciences, vol. 122, no. 4, pp. 661-672, 2012.

[2] T. Iwabuchi and R. Takada, "Time periodic solutions to the Navier-Stokes equations in the rotational framework," Journal of Evolution Equations, vol. 12, no. 4, pp. 985-1000, 2012.

[3] S. Ji and Y. Li, "Time periodic solutions to the one-dimensional nonlinear wave equation," Archive for Rational Mechanics and Analysis, vol. 199, no. 2, pp. 435-451, 2011. 
[4] E. A. Butcher, H. Ma, E. Bueler, V. Averina, and Z. Szabo, "Stability of linear time-periodic delay-differential equations via Chebyshev polynomials," International Journal for Numerical Methods in Engineering, vol. 59, no. 7, pp. 895-922, 2004.

[5] H. Hartono and A. H. P. Van Der Burgh, "A linear differential equation with a time-periodic damping coefficient: stability diagram and an application," Journal of Engineering Mathematics, vol. 49, no. 2, pp. 99-112, 2004.

[6] G. M. Amiraliyev and H. Duru, "A uniformly convergent difference method for the periodical boundary value problem," Computers and Mathematics with Applications, vol. 46, no. 5-6, pp. 695-703, 2003.

[7] Z. Cen, "Uniformly convergent second-order difference scheme for a singularly perturbed periodical boundary value problem," International Journal of Computer Mathematics, vol. 88, no. 1 , pp. 196-206, 2011.

[8] L. Peng-cheng and J. Ben-xian, "A singular perturbation problem for periodic boundary differential equation," Applied Mathematics and Mechanics, vol. 8, no. 10, pp. 929-937, 1987.

[9] G. M. Amiraliyev, Towards the Numerical Solution of the Periodical on Time Problem for Pseudo-Parabolic Equation, Numerical Methods of Analysis Baku State University, 1988.

[10] P. Giesl and H. Wendland, "Approximating the basin of attraction of time-periodic ODEs by meshless collocation of a Cauchy problem," Discrete and Continuous Dynamical Systems Supplements, pp. 259-268, 2009, 7th AIMS Conference.

[11] J. J. Hench and A. J. Laub, "Numerical solution of the discretetime periodic Riccati equation," IEEE Transactions on Automatic Control, vol. 39, no. 6, pp. 1197-1210, 1994.

[12] C. V. Pao, "Numerical methods for time-periodic solutions of nonlinear parabolic boundary value problems," SIAM Journal on Numerical Analysis, vol. 39, no. 2, pp. 647-667, 2002.

[13] E. V.Zemlyanaya and N. V. Alexeeva, "Numerical study of timeperiodic solitons in the damped-driven NLS," International Journal of Numerical Analysis \& Modeling, Series B, vol. 2, no. 2-3, pp. 248-261, 2011.

[14] G. M. Amiraliyev, "Difference method for a singularly perturbed initial value problem," Turkish Journal of Mathematics, vol. 22, no. 3, pp. 283-294, 1998.

[15] L. Herrmann, "Periodic solutions to a one-dimentional strongly nonlinear wave equation with strong dissipation," Czechoslovak Mathematical Journal, vol. 35, no. 2, pp. 278-293, 1985. 


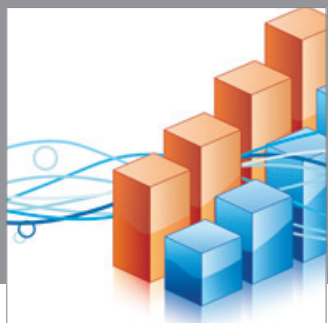

Advances in

Operations Research

mansans

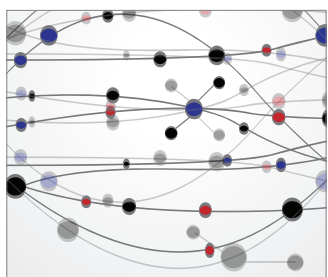

The Scientific World Journal
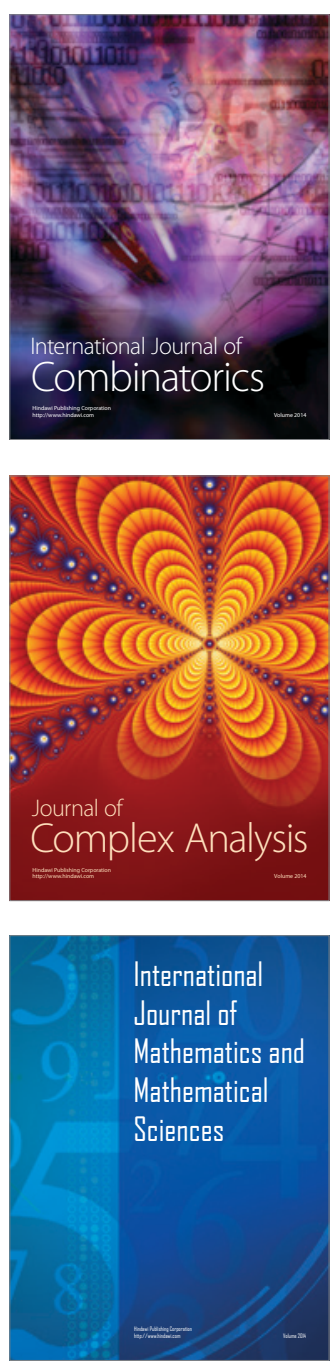
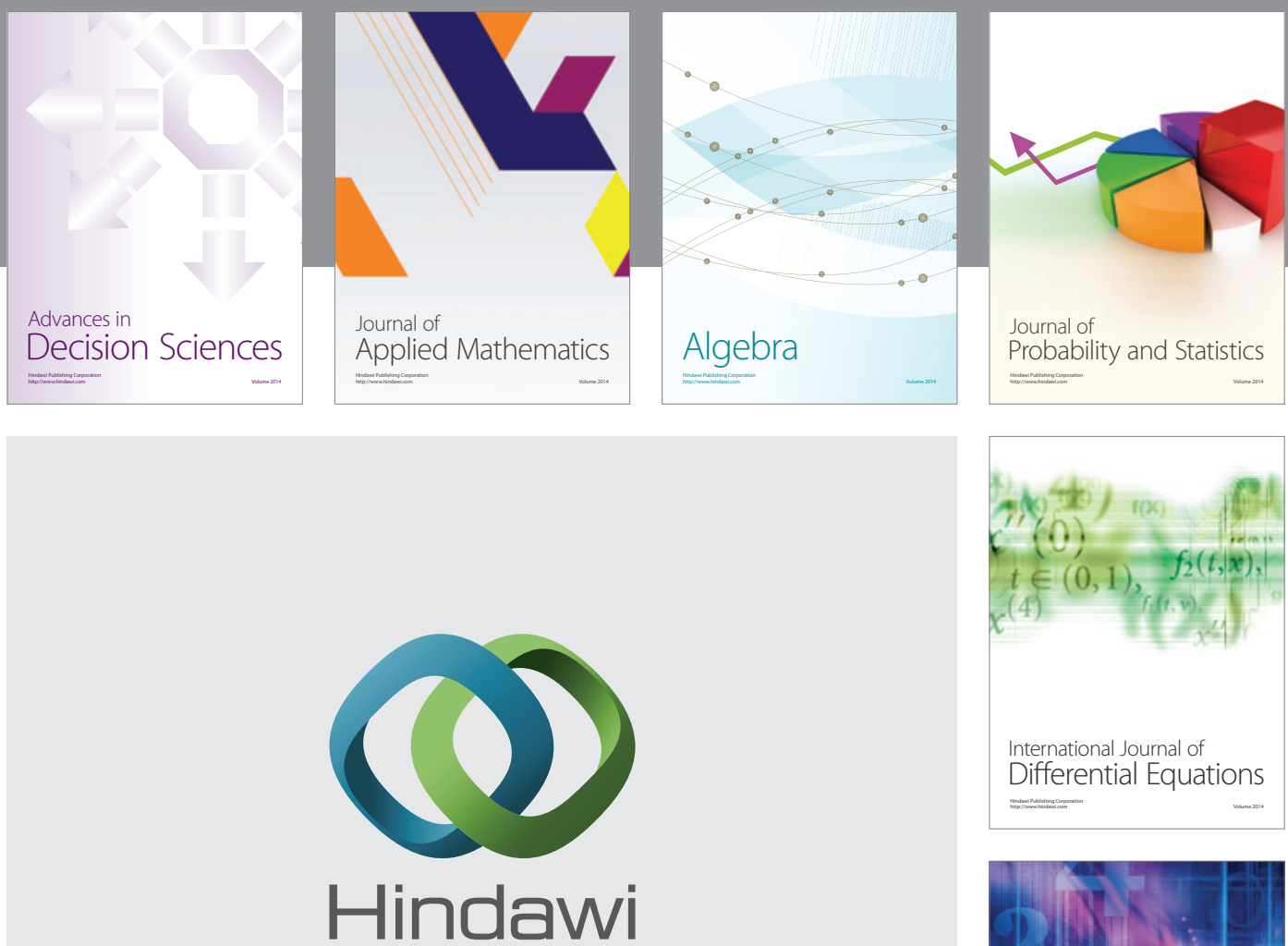

Submit your manuscripts at http://www.hindawi.com
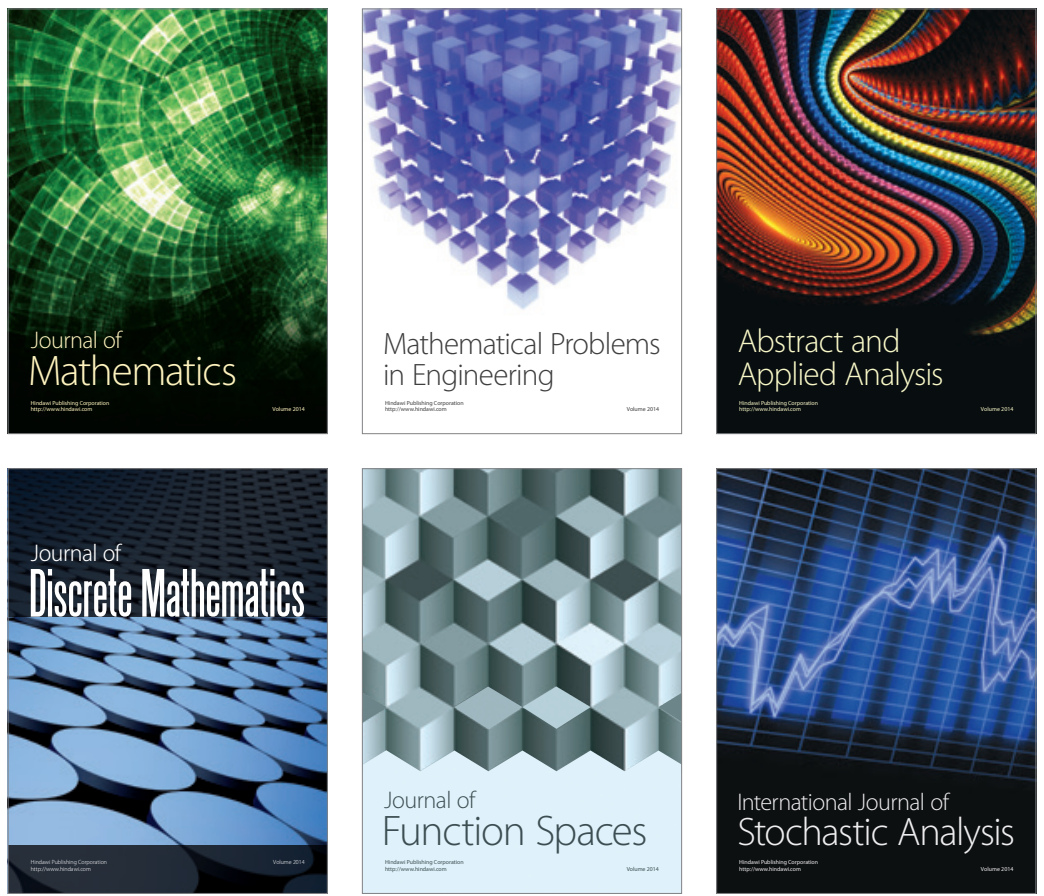

Journal of

Function Spaces

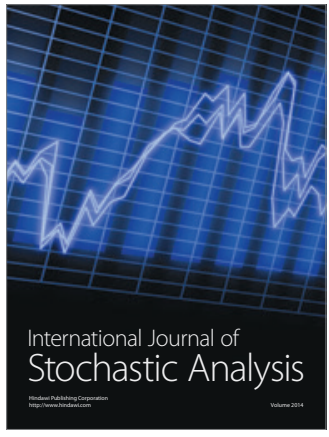

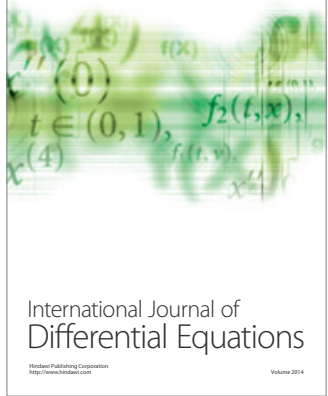
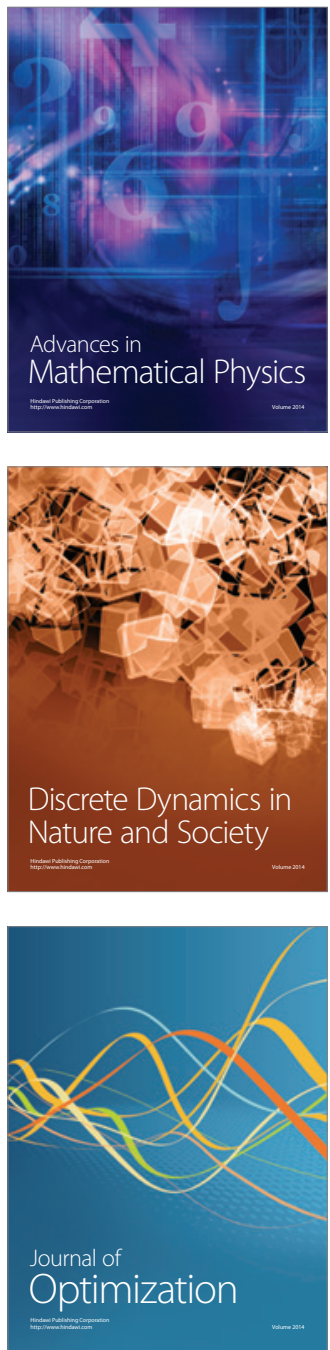